\title{
Erich Kunze 1905-1992
}

Prof., Prof. h.c., Dr. phil. Erich Kunze, geb. 30.4.1905 in Ottmachau (Schlesien), ist am 22.3.1992 in Göttingen gestorben, wohin er von Helsinki nach seiner Emeritierung im Jahre 1971 gezogen war. Mit dem Herzen hatte er Finnland nie verlassen, denn es war ihm zur geistigen Heimat geworden und auch in seinen Arbeiten, mit denen er sich während der Zeit des Ruhestandes beschäftigte, hielt er sich mehr in finnischen Gefilden auf, war er unterwegs zwischen Finnland und Deutschland.

Nach dem Studium der Germanistik, Geschichte und Philosophie in München, Heidelberg und Breslau (Promotion 1932) und der Tätigkeit als Universitätsassistent in Breslau 1932 kam er 1934 als Lektor für deutsche Sprache und Literatur nach Finnland, 1934-44 und 1947-52 nach Turku, 1952 nach Helsinki; seit 1958 (bis 1970) war er auch als Dozent der deutschen und vergleichenden Literaturgeschichte an der Universität Helsinki tätig. 1965-71 wirkte er als Professor für deutsche Sprache an der Handelshochschule in Helsinki; den Titel eines Prof. h.c. hatte er bereits 1961 verliehen bekommen.

Neben seiner Tätigkeit als Hochschullehrer hat Kunze ca. 4000 Seiten wissenschaftlicher Texte aus dem Finnischen ins Deutsche übersetzt oder auch Übersetzungen bearbeitet, häufig im Auftrag der Finnisch-Ugrischen Gesellschaft (z. B. die Publikationen der von Heikki Paasonen gesammelten Materialien aus der mordwinischen Volksdichtung, die von Paavo Ravila herausgegeben worden sind, vgl. MSFOu LXXVII, LXXXI, LXXXIV, XCI).

Verdienstvoll war seine Veröffentlichung einer Auswahl von Liedern aus Lönnrots Kanteletar; vor Kunze hatte nur Hermann Paul eine deutsche Übersetzung vorgelegt. Kunze hat die finnischen Lieder im Original und in deutscher Übersetzung herausgegeben (Kanteletar. Alte Volklieder und Balladen aus Finnland. Otava 1976).

Von unschätzbarer Wichtigkeit ist die Bibliographie, die Kunze erstmals 1950 publizierte ("Die deutschen Übersetzungen finnischer Schönliteratur") und die 1982 in erweiterter Form erschien unter dem Titel "Finnische Literatur in deutscher Übersetzung 1675-1975" (Helsingin yliopiston kirjaston julkaisuja 47). Es ist dies eine ausführliche, zuverlässige, mit großem Sachverstand eingeleitete und mit philologischer Akribie ausgearbeitete Bibliographie, eine Vorbedingung unter anderem für jegliche Rezeptionsforschung.

Besonders am Herzen lagen Erich Kunze die ideengeschichtlichen Verbindungen zwischen einzelnen Universitäten und Hochschullehren in Finnland 
und Deutschland; hierher gehört seine umfangreiche Untersuchung über die wissenschaftlichen Beziehungen zwischen der Göttinger Universität, der Georgia Augusta, und der Academia Aboensis (in: Gelehrte Kontakte zwischen Finnland und Göttingen zur Zeit der Aufklärung; 1988). Diese Publikation erschien in Göttingen im Zusammenhang mit einer dortigen Ausstellung aus Anlaß des 500jährigen Jubiläums des finnischen Buches.

Mit Umsicht und kritischer Sorgfalt hat Kunze Arbeiten geschaffen, die für die Geschichte der finnisch-deutschen literarischen, ideen- und geisteswissenschaftlichen Wechselbeziehungen wichtig sind. Seine diesbezüglichen Veröffentlichungen fügen sich zu einer inneren Einheit. Er veröffentlichte 1954 seine "Wirkungsgeschichte der finnischen Dichtung" (in: Deutsche Philologie im Aufriß, III); hier zeigte er, welche Wirkung die finnische Volksdichtung auf Grimm, Platen, Goethe, Rückert, Uhland, Karl Marx u.a. hatte. Kunze hat mit seinen Arbeiten zu einer neuen Dimension der Fennistik beigetragen, indem er die Erforschung der finnisch-deutschen literarischen und kulturellen $\mathrm{Be}$ ziehungen auf eine feste philologische Grundlage stellte. Mit ihm begann die eigentliche Rezeptionsforschung im finnisch-deutschen Bereich, er stellte sie in den ihr gemäßen europäischen Rahmen; nichts wäre dieser Fachrichtung mehr zu wünschen als Nachfolger, denn für die Erforschung dieser Kontakte bleibt noch viel zu tun.

Es ist ein besonderes Verdienst von Esko Häkli, Direktor der Universitätsbibliothek Helsinki und Herausgeber der Helsingin yliopiston kirjaston julkaisuja, $\mathrm{da} B$ er 1986 als Band 51 dieser Serie die wichtigsten, verstreut erschienenen Aufsätze von Erich Kunze zusammengefaßt und unter dem Titel "Deutsch-finnische Literaturbeziehungen. Beiträge zur Literatur- und Geistesgeschichte" publiziert hat. Kunze hat an 50 Arbeiten, Aufsätze und Rezensionen geschrieben, über die eine Bibliographie am Ende des genannten Bandes informiert. Viele seiner Arbeiten sind auch auf Finnisch erschienen, vor allem in dem Periodikum Kalevalaseuran vuosikirja und in der Serie Opusculum (beide Helsinki).

Kunze war bis in sein hohes Alter wissenschaftlich tätig. Sein letzter Aufsatz erschien 1992: "Frans Michael Franzén: Zur deutschen Rezeption des schwedischsprachigen Dichters Finnlands." (Journal of English and German Philology 91. 79-95.)

Erich Kunze war seit 1954 Auswärtiges Mitglied der Kalevalagesellschaft und seit 1971 Korrespondierendes Mitglied der Finnischen Literaturgesellschaft.

Sein Lebenswerk bleibt ein konstruktiver Beitrag zur Erforschung der mannigfachen Beziehungen zwischen Finnland und Deutschland im geistes- und literaturwissenschaftlichen Bereich. Dem aufrichtigen Finnlandfreund werden die, die ihn und seine Arbeiten kennen, ein ehrendes Andenken bewahren. 